\title{
Beschäftigungsperspektiven für Arbeitslose durch betriebsnahe und zielgruppenspezifische Qualifizierung
}

\begin{abstract}
Das Nachholen von fehlenden Qualifikationen ist ein wichtiger arbeitsmarktpolitischer Lösungsansatz zur Integration von Arbeitslosen in Beschäftigung. Dennoch wird dieses Instrument zu selten genutzt, da der direkte Nachweis der Beschäftigungswirksamkeit oft ausbleibt. Am Beispiel der „Qualifizierungsoffensive“ in der Seehafenwirtschaft stellt dieser Beitrag einen beschäftigungswirksamen Ansatz vor, bei dem Arbeitsagentur, Unternehmen und Gewerkschaft eng kooperieren. ${ }^{1}$ Im Vordergrund steht die Umschulung zur Fachkraft für Hafenlogistik. Diese Ausbildung in einen anerkannten Beruf ist für die Beantwortung der Fragen an eine gelingende Integration in Beschäftigung besonders interessant, da in einem erheblichen Maß Langzeitarbeitslose berücksichtigt werden sollten.
\end{abstract}

PETER MEHLIS, WILHELM EBERWEIN, JESSICA HEIBÜLT

\section{Problemhintergrund}

Das Thema Fachkräftemangel prägt in zunehmendem Maße die arbeitsmarktpolitische Debatte. Für einen erheblichen Mangel an Fachkräften gibt es empirisch - abgesehen von bestimmten Berufen und prosperierenden Regionen - zwar kaum Anzeichen, aber mit dem demografischen Wandel ist ein deutlicher Rückgang des Arbeitskräftepotenzials zu erwarten (Brenke 2010, S. 13; Dietz et al. 2012, S. 616). Insbesondere für Qualifizierte und Hochqualifizierte wird ein steigender Bedarf prognostiziert, während bei Geringerqualifizierten Beschäftigungseinbußen vorausgesagt werden. Um einen drohenden Fachkräftemangel abzuwenden, ist neben einer besseren Ausschöpfung des Arbeitskräftepotenzials die Erhöhung von Qualifikation und Produktivität der Arbeitskräfte nötig (Allmendinger/Ebner 2006, S. 230ff.; Fuchs et al. 2011, S. 7).

Die weiterhin hohe Zahl an Arbeitslosen verweist allerdings darauf, dass das vorhandene Arbeitskräftepotenzial einerseits nicht ausgeschöpft ist und andererseits Angebot und Nachfrage am Arbeitsmarkt häufig nicht zusammenpassen. Qualifizierung ist daher für die Integration von Arbeitslosen in Beschäftigung ein besonders wichtiges und, wie zahlreiche Studien zeigen, auch wirkungsvolles (Deeke et al. 2011, S. 201), aber nicht immer konsequent genutztes arbeitsmarktpolitisches Instrument.
Eine duale Berufsausbildung gilt als die beste Voraussetzung für einen raschen Übergang aus der Arbeitslosigkeit in Beschäftigung (Brixy/Christensen 2002; Klose/ Bender 2000; Bernhard et al. 2008). Doch gerade Leistungsbeziehern aus dem Rechtskreis des SGB II - mit dem überwiegenden Anteil der Langzeitarbeitslosen - mangelt es häufig an einer entsprechenden Ausbildung. Brenke sieht insbesondere in fehlenden Qualifikationen die Ursache für den schwächeren Rückgang der Arbeitslosigkeit bei den Hartz-IV-Empfängern trotz günstiger Beschäftigungsentwicklung (Brenke 2008, S. 684). Gelingt es Empfängern von Arbeitslosengeld II, eine Vollzeitbeschäftigung aufzunehmen, sind $44 \%$ von ihnen aufgrund niedriger Löhne auch weiterhin auf Leistungen aus dem Rechtskreis des SGB II angewiesen. Ferner erweisen

\footnotetext{
1 Das von der Hans-Böckler-Stiftung geförderte und vom Zentrum für Arbeit und Politik an der Universität Bremen durchgeführte Forschungsprojekt (Projektnr. 2009-318-5) untersuchte unter Berücksichtigung von arbeitsmarkt-, betriebs-, bildungs-, integrations- und transferpolitischen Fragestellungen die Qualifizierungsoffensive der Seehafenwirtschaft. In der Studie wurde aufgrund der differenzierten Fragestellungen und Zielgruppen ein Mix aus qualitativen und quantitativen Methoden verwendet. Neben Experteninterviews wurden schriftliche Befragungen der Qualifizierten durchgeführt und durch problemzentrierte Interviews (Witzel 1989) ergänzt. Die Studie ist in der edition sigma erschienen (Mehlis et al. 2013).
} 
sich die neuen Beschäftigungsverhältnisse als weniger dauerhaft: Nur $55 \%$ bestehen länger als sechs Monate (Koller/Rudolph 2011, S. 8).

Vor dem Hintergrund einer durch fehlende Qualifizierung verfestigten Langzeitarbeitslosigkeit bei gleichzeitig drohendem Fachkräftemangel ist es erstaunlich, dass die Anzahl der von der Bundesagentur für Arbeit (BA) finanzierten Maßnahmen im Rahmen des $₫ 77$ SGB III zur „Förderung beruflicher Weiterbildung“ (FbW), die $\mathrm{zu}$ einem Abschluss in einem anerkannten Beruf führen, zurückging. Im Jahr 2000 waren bundesweit 96.000 Eintritte in Maßnahmen mit Abschluss in einem anerkannten Beruf zu verzeichnen. 2008 waren es hingegen nur noch $26 \%$ der entsprechenden Eintritte des Jahres 2000 (Dietrich/Kruppe 2009, S. 262). Die Anzahl der Eintritte hat sich bis 2010 zwar wieder auf 52.100 erhöht (Statistisches Bundesamt 2011, S. 9), liegt aber immer noch deutlich unter dem Niveau von 2000. Dietrich und Kruppe halten die Möglichkeit, Arbeitslose durch berufliche Weiterbildung aus dem Bereich der Geringerqualifizierten herauszuführen, insgesamt für ausbaufähig (2009, S. 262). Langzeitarbeitslose waren zudem unter den Teilnehmern an FbW-Maßnahmen deutlich unterrepräsentiert (Bernhard et al. 2008).

Gleichzeitig wird aber auch deutlich, dass Qualifizierungen allein nicht zwangsläufig zu einer erfolgreichen Integration in Beschäftigung führen. Als erfolgreich erweisen sich vor allem betriebsnahe Qualifizierungsmaßnahmen, da hier Arbeitslose in den direkten Kontakt mit potenziellen Arbeitgebern treten. Die Orientierung an einem konkreten Arbeitskräfte- und Qualifizierungsbedarf steigert die Beschäftigungswirksamkeit der Qualifizierungsmaßnahmen. Gleichzeitig besteht allerdings die Gefahr von Mitnahmeeffekten seitens der Unternehmen (Koch et al. 2011, S. 3).

Festzuhalten ist, dass berufliche Weiterbildung als Mittel für eine gelingende Integration von Arbeitslosen mit fehlenden Qualifizierungen in Beschäftigung weitgehend alternativlos ist. Es stellt sich daher weniger die Frage, ob qualifiziert werden soll, sondern vielmehr wie eine erfolgreiche Qualifizierung und Integration in Beschäftigung aussehen können.

Im folgenden Abschnitt werden zunächst die Ausgangs- und Rahmenbedingungen der „Qualifizierungsoffensive" in der Seehafenwirtschaft sowie Art und Umfang der durchgeführten Maßnahmen referiert. Im Abschnitt 3 steht die Umschulung zur Fachkraft für Hafenlogistik als eine zentrale Qualifizierungsvariante im Mittelpunkt. Es geht um Fragen der Beschäftigungswirksamkeit, um den berücksichtigten Personenkreis und um förderliche Aspekte für eine gelingende Integration in Beschäftigung.

\section{Die „Qualifizierungsoffensive“}

\subsection{Ausgangssituation und Ziele}

Die Hafenwirtschaft erlebte bis Ende 2008 eine Boomphase mit teilweise zweistelligen Wachstumsraten. Mit der Expansion - insbesondere des Containerumschlags - entwickelten die deutschen Seehafenbetriebe eine starke Nachfrage nach Arbeitskräften. Gleichzeitig sind die Qualifikationsanforderungen an das Personal im modernen Hafenbetrieb gestiegen. Um einen entsprechenden Beschäftigungs- und Qualifikationsaufbau frühzeitig zu gestalten, hat der Zentralverband der Deutschen Seehafenbetriebe (ZDS) in Abstimmung mit der Gewerkschaft ver.di und in Zusammenarbeit mit dem Bundesministerium für Verkehr, Bau und Stadtentwicklung (BMVBS) und der Bundesagentur für Arbeit eine „Qualifizierungsoffensive" gestartet. Im Jahr 2006 wurde verabredet, dass der prognostizierte zusätzliche Bedarf von 2.800 Fachkräften bis 2012 durch die Qualifizierung und Einstellung von Arbeitslosen gesichert werden soll. Die Kosten für die Qualifizierung trägt die Agentur für Arbeit. Die Hafenwirtschaft hat den Teilnehmern ${ }^{2}$ bei erfolgreichem Abschluss der Qualifizierung die Übernahme in ein - tariflich abgesichertes - Beschäftigungsverhältnis garantiert (gemeinsame Pressemitteilung des ZDS, der Bundesagentur für Arbeit und des Bundesministeriums für Verkehr, Bau und Stadtentwicklung [BMVBS] vom 7.12.2007).

Der konjunkturelle Einbruch 2009 ist nicht spurlos an dem ambitionierten Vorhaben vorübergegangen. Die Prognosen bezüglich des zukünftigen Fachkräftebedarfs haben aber auch in der wirtschaftlichen Krise nicht an Bedeutung verloren. Allerdings wurden die Prioritäten in den Unternehmen neu definiert. Um die Krise zu überbrücken und gleichzeitig die Chancen langfristiger Wachstumsperspektiven mit der erwarteten konjunkturellen Erholung wahrnehmen zu können, machten einzelne Unternehmen der Hafenwirtschaft den gering qualifizierten Beschäftigten Qualifizierungsangebote im Zusammenhang mit konjunkturell bedingter Kurzarbeit. Mit diesen Angeboten für Stammbelegschaften wurde der Rahmen der „Qualifizierungsoffensive“ - Qualifizierungen von Arbeitslosen - erweitert. Die Gemeinsamkeit der Angebote bestand insbesondere in der Zielgruppe der gering qualifizierten Personen.

Die gemeinsam von wichtigen Akteuren des Arbeitsmarktes in Bremen/Bremerhaven, Wilhelmshaven, Ham-

2 Im vorliegenden Beitrag wird aus Gründen der Lesbarkeit i. d. R. auch bei nicht geschlechtsneutralen Bezeichnungen die männliche Form verwendet.

3 Als geringer qualifiziert werden Personen bezeichnet, die keinen beruflichen Abschluss haben oder in der letzten Beschäftigung Tätigkeiten ausübten, die unterhalb ihres Ausbildungsniveaus lagen und die damit die Fördervoraussetzungen des § 77 SGB III erfüllen. 
burg und Lübeck getragene „Qualifizierungsoffensive“ sowie die mit ihr intendierte Integration von Arbeitslosen und Geringerqualifizierten $^{3}$ in Ausbildung und Beschäftigung ist ein arbeitsmarktpolitischer Lösungsansatz, der für Benachteiligte am Arbeitsmarkt verspricht, im besonderen Maße beschäftigungswirksam zu sein. Die in der Hafenwirtschaft gemachten Erfahrungen und erzielten Ergebnisse haben daher arbeitsmarktpolitische Relevanz und verdienen eine genauere Betrachtung.

\subsection{Rahmenbedingungen}

Begünstigt wurden Entwicklung und Umsetzung der „Qualifizierungsoffensive" durch die engen und überschaubaren Kommunikations- und Kooperationszusammenhänge in der Hafenwirtschaft zwischen Unternehmen, Gewerkschaft und Agentur für Arbeit. Die Identifizierung von Schnittstellen gemeinsamer Interessen der beteiligten Akteure - als zentrale Voraussetzung für die Konzipierung und Implementation der "Qualifizierungsoffensive" - wurde so erleichtert. Dabei spielte die arbeitsmarktpolitisch nicht nur wünschenswerte, sondern bewusst intendierte und gestaltete wechselseitige Nutzenerwartung zwischen Arbeitsagentur und Unternehmen der Hafenwirtschaft eine zentrale Rolle. Die Beschäftigungszusage der Hafenbetriebe bewirkte ein weitreichendes finanzielles und personelles Engagement der Agentur.

Auch die Tarifvertragsparteien bewerteten die „Qualifizierungsoffensive" als eine Win-Win-Situation. Während Unternehmensleitungen in gut qualifizierten Beschäftigten einen wichtigen Vorteil der deutschen Seehäfen im europäischen Wettbewerb sehen - insbesondere durch eine höhere Flexibilität und Effektivität im Arbeitskräfteeinsatz -, verknüpft die Gewerkschaft ver.di mit einer zunehmenden Professionalisierung der Hafenarbeit eine langfristige Absicherung tariflicher Standards. Die Verknüpfung von ökonomischen Interessen der Unternehmen und gesellschaft- lichen Interessen am Abbau von Arbeitslosigkeit bescherte der Hafenwirtschaft zudem einen Imagegewinn.

Die gemeinsam von den Tarifvertragsparteien getragene Weiterbildungsstruktur in der Hafenwirtschaft hat zum Gelingen beigetragen. Ohne die Zusammenführung der regionalen hafenspezifischen Bildungseinrichtungen und die Gründung eines zentralen Bildungsträgers - das maritime competenzzentrum (ma-co) - wären Qualifizierungen in diesem Umfang und der Intensität kaum realisierbar gewesen. Zumal die fehlende Planungssicherheit infolge der Wirtschaftskrise und die fehlenden Erfahrungen mit der Qualifizierung im neuen Beruf Fachkraft für Hafenlogistik selbst das in der Hafenwirtschaft gut vernetzte ma-co vor Probleme stellten, die erst im Qualifizierungsprozess gelöst werden konnten. Dabei wird deutlich, dass eine Qualifizierung „on demand“ schnell auf organisatorische und inhaltliche Grenzen stoßen kann, die sich nur unter erheblichem Einsatz und mit entsprechenden Ressourcen überwinden lassen.

\subsection{Art und Umfang der Qualifizierungen}

Traditionell wurden Arbeitskräfte für den Hafen aus anderen Wirtschaftsbereichen mit der Aussicht auf eine bessere Entlohnung abgeworben. Die Studie zur Hafenarbeit von Abendroth et al. von 1979 stellte fest, dass der überwiegende Teil der Hafenarbeiter (80 \%) über einen Berufsabschluss verfügte, der allerdings wenig mit Hafenarbeit zu tun hatte. Die Tätigkeit war demnach Anlernarbeit, die auf einer berufsfremden Qualifikation aufbaute. Qualifizierung wurde als Anpassungsprozess verstanden, der auf konkretem Erfahrungswissen in den Einsatzbereichen und begleitenden Lehrgängen beruhte (Abendroth et al. 1979, S. 210f.). Der Zugang zur Hafenarbeit war nur in einem geringen Ausmaß durch Barrieren in Form von Qualifikationsnachweisen geprägt und wies zudem beim Erwerb weiterer hafenspezifischer Qualifikationen eine große Durchlässigkeit auf,

TABELLE 1

Mit öffentlichen Mitteln durchgeführte Qualifizierungen an den Hafenstandorten Hamburg, Bremerhaven, Wilhelmshaven und Lübeck

Angaben in absoluten Zahlen

\begin{tabular}{|c|c|c|}
\hline & Qualifizierung von Arbeitslosen ${ }^{1}$ & $\begin{array}{c}\text { Weiterqualifizierung von Beschäftigten in } \\
\text { Unterbeschäftigung }{ }^{2}\end{array}$ \\
\hline Fachkraft Containerumschlag & 1.816 & \\
\hline Hafenfacharbeiter & & 123 \\
\hline Fachkraft für Hafenlogistik & 308 & 198 \\
\hline diverse andere Qualifizierungen & & 359 \\
\hline Gesamt & 2.124 & 680 \\
\hline
\end{tabular}

${ }^{1}$ Im Rahmen des $\S 77$ Abs. 1 SGB II. ${ }^{2}$ Im Rahmen der Qualifizierung von Kurzarbeitenden (§ 77 Abs. 2 SGB III und ESF) sowie des WeGebAU-Programms. 
die es auch Geringerqualifizierten im Verlauf des Erwerbslebens ermöglichte, ihren beruflichen Status zu erweitern. Diese traditionellen Rekrutierungs- und Qualifikationswege haben sich im Kern bis heute erhalten, werden aber dem technischen und organisatorischen Wandel in der Hafenarbeit zunehmend angepasst. Insgesamt lässt sich von einer Professionalisierung der Hafenarbeit durch eine Formalisierung und Systematisierung sprechen, die den gestiegenen Anforderungen entspricht und mit einer Höherqualifizierung der Beschäftigten verbunden ist.

Im Rahmen der „Qualifizierungsoffensive" lassen sich vier essenzielle Qualifizierungsmaßnahmen identifizieren (Tabelle 1):

- Eine dreimonatige Basisqualifizierung von Arbeitslosen zur Fachkraft Containerumschlag ( $\mathrm{FaCo}$ ) soll die Teilnehmer/innen für die Arbeit auf dem Terminal sowie verschiedene Funktionen des Containerumschlags qualifizieren.

- Eine 24-monatige Umschulung von Arbeitslosen zur Fachkraft für Hafenlogistik. Der staatlich anerkannte Ausbildungsberuf wurde 2006 neu geschaffen; in der Ausbildung werden umfassende Kenntnisse über die logistischen Prozesse im Hafen vermittelt.

- Eine dreimonatige Anpassungsqualifizierung von geringer qualifizierten Beschäftigten zum Hafenfacharbeiter im Rahmen von Kurzarbeit. Der Hafenfacharbeiter ist ein anerkanntes Berufsbild der beruflichen Fortbildung gemäß $₫ 53$ des Berufsbildungsgesetzes.

- Eine neunmonatige Anpassungsqualifizierung von geringer qualifizierten Beschäftigten zur Fachkraft für Hafenlogistik im Rahmen von Kurzarbeit und des Programms Weiterbildung Geringqualifizierter und beschäftigter älterer Arbeitnehmer in Unternehmen (WeGebAU).

Zudem wurden im Rahmen von Kurzarbeit und WeGebAUProgramm weitere diverse Qualifizierungen angeboten und durchgeführt.

Insbesondere in der Boomphase benötigten die Hafenbetriebe möglichst schnell eine größere Anzahl von Arbeitskräften, sodass überwiegend für den kurzfristigen Bedarf in drei Monaten zur Fachkraft Containerumschlag ausgebildet wurde. Arbeitslose, die erfolgreich die FaCo-Qualifizierung absolvierten, erhielten einen unbefristeten Arbeitsvertrag. ${ }^{4}$ Mit dem massiven Rückgang des Hafenumschlags 2008/2009 wurden diese kurzfristigen Qualifizierungen vorübergehend eingestellt. Die Firmenpolitik bestand nunmehr darin, den Personalstand stabil zu halten und die Unterauslastung durch Kurzarbeit zu überbrücken. Um rechtzeitig mit der Wiederbelebung der Exportwirtschaft über Fachkräfte zu verfügen, verfolgten einige Hafenbetriebe die Strategie, geringer qualifizierte Beschäftigte in der Krise in Verbindung mit Kurzarbeit zu qualifizieren. Die Angebote sind überwiegend Beschäftigten zugutegekommen, die im Rahmen einer Kurzqualifizierung zur Fachkraft Containerumschlag eingestellt wurden und nun zum Hafenfacharbeiter oder zur Fachkraft für Hafenlogistik weitergebildet wurden.
Eine Besonderheit bilden die Qualifizierungen für den im September 2012 neu eröffneten Tiefwasserhafen in Wilhelmshaven. Da ein größeres Zeitfenster bis zur Inbetriebnahme bestand und eine qualifizierte Belegschaft den reibungslosen Start ermöglichen sollte, wurden seit 2008 in mehreren Wellen Arbeitslose innerhalb von zwei Jahren zur Fachkraft für Hafenlogistik umgeschult. Analog zu diesen Maßnahmen wurden seit 2010 auch in Lübeck Arbeitslose zur Fachkraft für Hafenlogistik ausgebildet, um den langfristigen Fachkräftebedarf zu decken.

Die gestaltenden Akteure - Hafenbetriebe, Agentur für Arbeit, Bildungsträger - haben insgesamt ein hohes Maß an Flexibilität gezeigt, indem sie die Qualifizierungsziele an die jeweiligen wirtschaftlichen Rahmenbedingungen anpassten und geschickt die sich öffnenden Zeitfenster für Qualifizierungen nutzten.

Seit 2008 waren die langfristig angelegten Qualifizierungen - die 24-monatige Umschulung und die neunmonatige Anpassungsqualifizierung zur Fachkraft für Hafenlogistik die tragenden Säulen der „Qualifizierungsoffensive“.

\section{Die Umschulung zur Fachkraft für Hafenlogistik}

Die im Rahmen der "Qualifizierungsoffensive“ durchgeführte Umschulung zur Fachkraft für Hafenlogistik eröffnete den teilnehmenden Arbeitslosen nachhaltige Beschäftigungsperspektiven. Mit dem erfolgreichen Abschluss wurde den Teilnehmer/inne/n die Übernahme in ein unbefristetes, tariflich abgesichertes Beschäftigungsverhältnis zugesagt. Hohe Löhne, tarifliche Standards, vielfältige Weiterqualifizierungsangebote und eine starke Interessenvertretung der Arbeitnehmer/innen verweisen auf attraktive und stabile Beschäftigungsverhältnisse, die bislang selbst schwierige konjunkturelle Phasen überstanden haben. Außerdem reicht die Qualifizierung weit über eine Teilqualifikation, die nahe am betrieblichen Bedarf orientiert ist, hinaus. Eine Verbesserung der individuellen Arbeitsmarktchancen besteht somit auch über den ausbildenden Betrieb hinaus. Da mit dem Umschulungsangebot zudem insbesondere Langzeitarbeitslose angesprochen werden sollten, wird im Folgenden diese Qualifizierungsvariante näher betrachtet, um wichtige Gestaltungs- und Erfolgsmerkmale hervorzuheben.

\footnotetext{
4 Alle Bewerber für die FaCo-Qualifizierung durchliefen zunächst ein Auswahlverfahren. Die Auswahlquote betrug etwa $50 \%$. Mit festgestellter Eignung bekamen dieTeilnehmer/innen der Qualifizierung gleichzeitig die Einstellungszusage bei erfolgreichem Abschluss, der nahezu allen gelang. Die geringe Fluktuation in der Hafenwirtschaft und die Überbrückung der Krise durch Kurzarbeit führten zu einem dauerhaften Beschäftigungsaufbau.
} 


\subsection{Leitgedanken}

Die Gründung des neuen Tiefwasserhafens in Wilhelmshaven gleichsam „auf der grünen Wiese“ stellte eine besondere Herausforderung dar, galt es doch, damit gleichzeitig eine komplett neue Belegschaft aufzubauen. In der Startphase konnte und wollte der Hafenbetrieb die traditionelle Rekrutierungspraxis, die Abwerbung von Arbeitskräften aus anderen Betrieben und sukzessive aufeinander aufbauende Kurzqualifikationen, nicht nutzen. Vielmehr ging es darum, den neuen Standort von Beginn an mit einer qualifizierten und voll einsatzfähigen Kernbelegschaft betreiben zu können. Die problematische Situation am Arbeitsmarkt in Wilhelmshaven bedeutete zugleich, dass ein überdurchschnittlicher Anteil von Langzeitarbeitslosen berücksichtigt werden musste. Vor diesem Hintergrund strebten die Akteure - Hafenbetrieb und Agentur für Arbeit - einen Anteil von 75 \% Langzeitarbeitslosen bei der Umschulung an.

Durch die Übernahme der Qualifizierungskosten und der Transferleistungen für den Lebensunterhalt der Umschüler durch die Agentur für Arbeit bzw. die Jobcenter wurde das Unternehmen zwar finanziell erheblich entlastet, aber es bestanden beträchtliche Unsicherheiten, ob die zukünftigen Mitarbeiter die in sie gesetzten Erwartungen erfüllen und einen reibungslosen Start des neuen Tiefwasserhafens würden gewährleisten können. Auch in den Agenturen bestand ein Interesse, nur solche Kandidaten zu benennen, die in der Lage sind, die Qualifizierung erfolgreich abzuschließen. Um die Risiken des Scheiterns zu minimieren, wurden daher drei zentrale Kriterien in das Qualifizierungskonzept eingebaut:

- In einem differenzierten Auswahlprozess wurde die Eignung potenzieller Umschüler für die Qualifizierung in der Hafenlogistik festgestellt.

- Mit einer zielgruppengerechten Gestaltung der theoretischen und praktischen Ausbildung wurde angestrebt, alle ausgewählten Teilnehmer zu einem erfolgreichen Abschluss zu führen.

- Die erfolgreiche Integration der Umschüler in Ausbildung und Beschäftigung sollte durch sozialpädagogische Begleitung und Weiterbildungsberatung unterstützt werden. Ferner sollte das Unternehmen bei der Organisation und Durchführung der Qualifizierung beraten werden.

\subsection{Auswahlprozess und Sozialprofil}

Für die Umschulung wurde ein dreistufiges Auswahlverfahren entwickelt. Die Arbeitsagentur identifizierte zunächst anhand einer Checkliste potenzielle Kandidaten, die in einer ersten Stufe vom Psychologischen Dienst der Agentur bezüglich ihrer grundsätzlichen Eignung mittels eines standardisierten Tests und eines individuellen Gesprächs (Themen waren Motivation, Hafentauglichkeit und Lebenslauf) überprüft wurden. Geeignete Kandidaten nahmen in einer zweiten Stufe an einer einwöchigen Trainingseinheit (TM 1) teil. Ein externer Bildungsträger führte mit den Teilnehmern u. a. einen Berufseignungstest, einen Konzentrationstest und einen Theorietest durch. Kandidaten, die die TM 1 erfolgreich abgeschlossen hatten, nahmen an einer weiteren siebentägigen Trainingseinheit (TM 2) teil. Diese wurde gemeinsam von dem beteiligten Hafenbetrieb und dem ma-co durchgeführt.

Für die Vertreter des Hafenbetriebes hatten insbesondere die Sozialkompetenzen der Umschüler einen wichtigen Stellenwert im Auswahlverfahren. Die Fähigkeit zur Teamarbeit, die Motivation für eine Tätigkeit im Hafenumschlag und die Verlässlichkeit sind wichtige Attribute, die das Unternehmen von zukünftigen Mitarbeitern und Mitarbeiterinnen erwartet und die in Gruppenübungen und Planspielen beurteilt wurden. Die endgültige Entscheidung über eine Teilnahme an der Umschulung wurde vom Unternehmen gefällt, da mit der Zusage für die Umschulung auch eine Übernahme in ein unbefristetes Arbeitsverhältnis bei erfolgreichem Abschluss verknüpft war und ein langfristiges Beschäftigungsverhältnis angestrebt wurde.

Das aufwendige Auswahlverfahren hat zu einer erheblichen Selektion bei dem zunächst vorgeschlagenen Personenkreis geführt, sodass man durchaus von einer relativen „Bestenauswahl“ sprechen kann. Die Auswahlquote lag bei $19 \%$. Auffällig war auch, dass sich das Verhältnis von Bewerber im ALG I-Bezug (SGB III) zu denen im ALG-IIBezug (SGB II) im Verlauf des Auswahlverfahrens umgekehrt hat und von den letztendlich als geeignet eingestuften Personen deutlich mehr ALG I bezogen. Somit sind im Auswahlprozess überdurchschnittlich oft Langzeitarbeitslose - in der Regel Personen im ALG-II-Bezug (SGB II) ausgeschieden; der ursprünglich angestrebte Anteil von $75 \%$ wurde nicht erreicht. Der tatsächliche Anteil der Teilnehmer im ALG-II-Bezug, der in die Umschulung einmündete, betrug $42 \%$.

Das Ziel, einen Frauenanteil von 10 \% zu erreichen, wurde knapp verfehlt. Hier zeigt sich, dass Hafenarbeit als Beschäftigung für Frauen erst am Anfang steht und bei diesen noch an Akzeptanz gewinnen muss. Insbesondere eine gelingende Work-Life-Balance dürfte für Frauen angesichts der im Hafen üblichen Schicht- und Wochenendarbeit schwerer zu erreichen sein als für Männer. Die von den Hafenbetrieben angestrebte Erhöhung des Frauenanteils ist daher eng mit entsprechenden Work-Life-Balance-Angeboten zu verknüpfen.

Die Sozialstruktur der insgesamt 260 Umschüler war hinsichtlich Alter, Familienstand, Schulbildung und Berufsabschlüssen sehr heterogen. Die Verteilung nach Alter war relativ ausgeglichen und eine Benachteiligung einer Alterskohorte - wenn man von der Gruppe der Über-52-Jährigen absieht - nicht erkennbar. Hauptschul- (40\%) und Realschulabschlüsse (45\%) dominierten, immerhin $15 \%$ der Umschüler verfügten über einen höheren Schulabschluss. $22 \%$ der Teilnehmer hatten bislang keinerlei berufliche Ausbildung genossen. 
Trotz der geringeren Anzahl von Umschülern im ALGII-Bezug ist durch das Auswahlverfahren der ursprüngliche Fokus auf die sogenannten Problemgruppen des Arbeitsmarktes keineswegs verfehlt worden. Verallgemeinernd konnten wir in unserer Untersuchung zwei verbreitete Muster bei den Teilnehmern ausmachen. Diese schlagen sich zum einen in Erwerbsbiografien nieder, die deutliche Dequalifizierungsprozesse aufweisen und zum anderen in Erwerbsbiografien, die durch fehlende berufliche Qualifikationen gekennzeichnet sind. Für beide Muster ist charakteristisch, dass überwiegend Anlerntätigkeiten ausgeübt wurden, die häufig schlecht bezahlt wurden und befristet waren. Die Beschäftigungsphasen wurden häufig durch Zeiten der Arbeitslosigkeit unterbrochen. Der überwiegende Teil der in unserem Sample erfassten Arbeitslosen hatte eine vergleichbare Erwerbskarriere durchlebt. Insofern gehören viele Umschüler zwar nicht formal, aber doch inhaltlich zu der durch die "Qualifizierungsoffensive“ angestrebten Klientel. Teilnehmer mit den beiden beschriebenen Mustern der Erwerbsbiografie konnten mit der Umschulung Dequalifizierungsprozesse umkehren bzw. fehlende Qualifikationen nachholen und eine nachhaltige Integration in eine unbefristete und tariflich abgesicherte Beschäftigung erreichen.

\subsection{Spezifische Gestaltung und flankierende Unterstützung}

Unsere Befragungen der Umschüler zeigten: Die Aussicht auf sichere Beschäftigung und guten Verdienst, verbunden mit interessanten Arbeitsinhalten, attraktiven Arbeitsbedingungen und kollegialen Solidarstrukturen bildeten die zentrale Grundlage für die hohe Motivation der Umschüler,

ABB. 1

Relative Häufigkeit der Beratungsbedarfe nach Themen und Rechtskreisen* Angaben in Prozent - SGB III SGB II

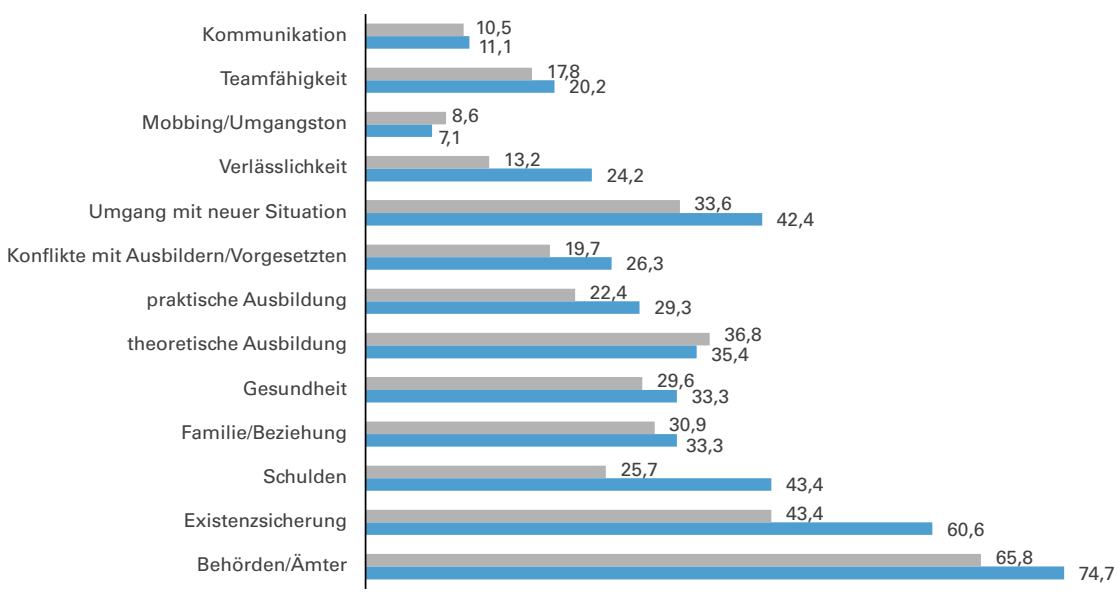

${ }^{*} \mathrm{~N}=260$

Quelle: Daten der Autoren.

Mitteilungen die ihrerseits eine langfristige Bindung an das Unternehmen anstreben.

Die von Beginn an angestrebte zielgruppenspezifische Ausbildung hat sich als notwendig erwiesen und - nach Startschwierigkeiten - bewährt. Lernförderlich waren insbesondere die Ausbildung durch Praktiker, die insgesamt positive Lernsituation in der Gruppe, aber auch die spezifische Arbeitskultur des Hafens sowie der Versuch, durch eine enge Lernortkooperation Theorie und Praxis wechselseitig aufeinander zu beziehen und das Bestreben, möglichst alle Teilnehmer „mitzunehmen“.

Schließlich ist auch die Flankierung der Umschulung Beratung und Begleitung der Umschüler und des beteiligten Unternehmens durch ein eigens installiertes Projekt „Hafenlogistik - Bleib dran“ ein weiteres Element der zielgruppenspezifischen Qualifizierung, da der differenzierte Auswahlprozess und die hohe Motivation der Teilnehmer eine zwar notwendige, aber noch keine hinreichende Garantie für den Ausbildungserfolg erwarten ließen. Das beteiligte Unternehmen stellte daher für die flankierende Unterstützung die finanziellen Mittel bereit.

Das Projekt konnte an Erfahrungen in der dualen Berufsausbildung und ein dort entwickeltes Konzept zur Prävention von und Intervention bei Konflikten in der Ausbildung anknüpfen (Quante-Brandt 2003; MahlbergWilson et al. 2009). Als Leitlinien der Arbeit gelten Allparteilichkeit (Neutralität), persönliche Verschwiegenheit und strukturelle Offenheit, d. h. strukturelle Erkenntnisse, die in der Begleitung der Umschüler gewonnen werden, können im Sinne einer Prozessoptimierung genutzt werden.

Bei der betroffenen Klientel der Arbeitslosen findet sich oft eine Gemengelage an Problemen und kritischen Lebenssituationen, die sie alleine nicht bewältigen können und die den Ausbildungserfolg bedrohen. Insbesondere finanzielle Probleme infolge langer Arbeitslosigkeit und niedriger Einkommen, Konflikte mit Ämtern und familiäre Probleme bilden neben den Schwierigkeiten mit der (Wieder-)Aufnahme des Lernprozesses das Substrat für alltäglich auftretende Probleme und Konflikte. Das Unterstützungsangebot für die Umschüler bezog sich nicht nur auf eine Lern- und Bildungsberatung, sondern bot bei Bedarf auch eine sozialpädagogische Begleitung (Abbildung 1).

In der Untersuchung lassen sich bezüglich der Beratung und Begleitung vier Nutzungsprofile bei den Umschülern unterscheiden:

- Personen, die keinen Bedarf haben und ihre Dinge selber regeln;

- Personen ohne zwingenden Bedarf, die aber in dem Projekt „Hafenlogistik - Bleib dran“ eine nützliche Einrichtung sehen und das gut vernetzte Beratungsangebot als „Vermittlungsund Informationsstelle" nutzen;

- Personen, die bei Lern- und Lebensfragen Beratung benötigen und bei denen die Gefahr des Scheiterns ohne Begleitung groß ist; 
- Personen, welche Beratung nötig haben, dies aber als persönliche Schwäche empfinden und daher zunächst Beratungsbedarf verneinen. Erst in eskalierenden Situationen nimmt die Bereitschaft zu, sich für das Beratungsangebot zu öffnen.

Die Beratungsstatistik zeigt, dass beinahe 85 \% der Umschüler das Unterstützungsangebot aus eigenem Antrieb in Anspruch nahmen.

Ein weiterer Arbeitsschwerpunkt war die Beratung des Unternehmens bei der Gestaltung und Organisation der Umschulung. Hierzu gehören die Beratung im Auswahlverfahren, das Coaching der betrieblichen Ausbilder, Beteiligung an der Prozessoptimierung der Umschulung und das Schnittstellenmanagement. Die Befunde zeigen, dass „Hafenlogistik - Bleib dran im Qualifizierungsnetzwerk eine eminent wichtige kommunikative und intermediäre Rolle ausfüllte. Es sprang überall da ein, wo der Informationsfluss stockte und hielt damit das alltägliche Umschulungsgeschehen am Laufen. Vertreter von Unternehmen und Agentur für Arbeit bewerteten gleichermaßen das Projekt als sehr wertvoll, da sie bei der Lösung zahlreicher Probleme und Konflikte entlastet wurden.

\subsection{Umschulungsergebnisse und Verbleib der Qualifizierten}

Alle Umschulungswellen haben die Qualifizierung zur Fachkraft für Hafenlogistik abgeschlossen. Die letzte Welle folgte im Januar 2013. Es lassen sich somit für alle 260 Umschüler Aussagen zum Qualifizierungserfolg und Verbleib machen. 244 Umschüler haben die Prüfung bestanden, 238 haben einen unbefristeten Arbeitsvertrag unterschrieben. Die Integration in Beschäftigung durch Qualifizierung kommt auf eine Erfolgsquote von $92 \%$. Die Quote gemindert haben lediglich Umschulungsabbrüche. Im Vergleich zur dualen Berufsausbildung bzw. den Umschulungen von Arbeitslosen ohne direkte betriebliche Anbindung fällt die Abbruchquote mit $5 \%$ (13) aber sehr moderat aus. Eine vergleichbar niedrige Abbruchquote ist an sonsten nur in der dualen Ausbildung von Berufen zu beobachten, die aufgrund ihrer Attraktivität, guten Ausbildungsbedingungen und der Bewerberauswahl geringe Probleme mit Ausbildungsabbrüchen haben (Bundesinstitut für Berufsbildung 2011, S. 186).

\section{Fazit}

Die gemeinsam von Agentur für Arbeit, Hafenwirtschaft und Gewerkschaft getragene "Qualifizierungsoffensive“ ist ein besonders wirksamer arbeitsmarktpolitischer Lösungsansatz, Arbeitslose und Geringerqualifizierte in Ausbildung und Beschäftigung zu integrieren. Vor dem Hintergrund der zwischenzeitlich kritischen wirtschaftlichen Entwicklung in Folge der Finanzkrise 2008 erscheint die Anzahl von etwa 2.000 qualifizierten und eingestellten Arbeitslosen aus arbeitsmarktpolitischer Perspektive mehr als akzeptabel. Zudem wurden in der Krise auf Entlassungen verzichtet und weitere Qualifizierungen für etwa 680 überwiegend geringer qualifizierte Beschäftigte in Verbindung mit Kurzarbeit durchgeführt. Bestehende Beschäftigungsverhältnisse wurden somit aufgewertet sowie zukunftsfähiger gestaltet.

Hervorzuheben ist die zeit- und kostenintensive Umschulung zur Fachkraft für Hafenlogistik. Gut ausgebildetes Personal stärkt nicht nur die Wettbewerbsfähigkeit der Hafenbetriebe, sondern bietet den ehemals Arbeitslosen stabile, tariflich abgesicherte Beschäftigung. Die Bereitschaft der
Betriebe, diesen Weg zu gehen, wurde nicht nur durch finanzielle Anreize der Agentur beeinflusst. Das differenzierte Auswahlverfahren und die flankierende Unterstützung haben dazu beigetragen, Risiken kalkulierbar zu machen und die Entscheidung der Unternehmen für diesen Weg zu ebnen. Die Erfolgsquote - $92 \%$ der anfänglich gestarteten 260 Umschüler haben die Ausbildung erfolgreich absolviert und wurden in ein unbefristetes Beschäftigungsverhältnis übernommen - zeigt sehr deutlich, dass es gelungen ist, die Risiken zu minimieren.

Trotz des geringer als ursprünglich intendierten Anteils an Langzeitarbeitslosen wurde die arbeitsmarktpolitische Intention mit der getroffenen Auswahl nicht verfehlt. Allerdings macht das Beispiel der großen Anzahl abgelehnter Bewerber auch deutlich, dass insbesondere für den Personenkreis der am Arbeitsmarkt Benachteiligten weiterhin Handlungsbedarf besteht.

Insgesamt haben die Rahmenbedingungen in der Hafenwirtschaft - langfristige wirtschaftliche Zukunftsperspektive, nachhaltige Personalentwicklungsstrategie, Akteurskonstellation und entwickelte Weiterbildungsinfrastruktur - die Durchführung einer „Qualifizierungsoffensive“ begünstigt. Die genannten Aspekte sind allerdings kein Alleinstellungsmerkmal der Hafenwirtschaft und eine Übertragung des Qualifizierungs- und Beschäftigungskonzepts in andere Branchen/Unternehmen scheint keinesfalls ausgeschlossen, wenn Qualifizierungs- und Arbeitskräftebedarfe festgestellt werden. Die betriebsnahe - an konkreten Arbeitskräfte- und Weiterbildungsbedarfen orientierte - Qualifizierung ist nachvollziehbar beschäftigungswirksam und zeigt einen Weg auf, wie Weiterbildung besonders effektiv als arbeitsmarktpolitisches Instrument genutzt werden kann. Die Hafenwirtschaft wird in ihrem Handeln primär von betriebswirtschaftlichen Interessen geleitet, was die Gefahr von Mitnahmeeffekten erhöht. Die „Qualifizierungsoffensive“ machte deutlich, dass in einem permanenten Aushandlungsprozess zwischen den beteiligten Akteuren eine Win-Win-Situation hergestellt werden konnte.

Insbesondere das flankierende Unterstützungsangebot ist ein innovativer Bestandteil der Umschulung, das nicht nur hilft, einem Scheitern der Individuen in der Qualifizierung vorzubeugen, sondern insgesamt dazu beitragen kann, die Bereitschaft der Betriebe zur Qualifizierung und Einstellung vermeintlicher Problemgruppen des Arbeitsmarktes zu befördern. Unsere Befunde zeigen, dass ein erfolgreiches Unterstützungsangebot nicht auf sozialpädagogische Betreuung der Arbeitslosen beschränkt bleiben darf, sondern eine insgesamt die Qualifizierung tragende Rolle als Schnittstellenmanager und beratende Instanz für alle beteiligten Akteure einnehmen muss. Insbesondere Unternehmen und Arbeitsagentur werden durch Nutzung externen Fachwissens und durch „Auslagerung“ zahlreicher Problemlagen erheblich entlastet.

\section{LITERATUR}

Abendroth, M./Beckenbach, N./Braun, S./Dombois, R. (1979): Hafenarbeit. Eine industriesoziologische Untersuchung der Arbeits- und Betriebsverhältnisse in den bremischen Häfen, Frankfurt a. M./New York

Allmendinger, J./Ebner, C. (2006): Arbeitsmarkt und demografischer Wandel, in: Zeitschrift für Arbeits- und Organisationspsychologie 50 (4), S. 227-239 Bernhard, S./Hohmeyer, K./Jozwiak, E./Koch, S./Kruppe, T./Stephan, G./Wolff, J. (2008): Aktive Arbeitsmarktpolitik in Deutschland und ihre Wirkungen, IABForschungsbericht 2/2008, Nürnberg

Brenke, K. (2008): Arbeitslose Hartz IV-Empfänger: Oftmals gering qualifiziert, aber nicht weniger arbeitswillig, in: DIW-Wochenbericht 75 (43), S. 678-684, Berlin

Brenke, K. (2010): Fachkräftemangel kurzfristig noch nicht in Sicht, in: DIW-Wochenbericht 77 (46), S. 2-15, Berlin 
Brixy, U./Christensen, B. (2002): Flexibilität - Wie viel würden Arbeitslose für einen Arbeitsplatz in Kauf nehmen?, IAB-Kurzbericht 25/2002, Nürnberg Bundesinstitut für Berufsbildung (BIBB) (2011): Datenreport zum Berufsbildungsbericht 2010, Bonn

Deeke, A./Dietrich, H./Kruppe,T./Lott, M./Rauch, A./Stephan, G./Wolf, J.

(2011): Geförderte Qualifizierungsmaßnahmen in Deutschland: Aktuelle Evaluationsergebnisse im Überblick, in: Sozialer Fortschritt 60 (9), S. 196-203

Dietrich, H./Kruppe, T. (2009): Qualifizierung im Erwerbsverlauf - Eine Chance in der Wirtschaftskrise, in: Sozialer Fortschritt 58 (11), S. 257-264

Dietz, M./Kubis, A./Müller, A. (2012): Fachkräftemangel in Ost- und Westdeutschland? Eine betriebliche Perspektive, in: WSI-Mitteilungen 65 (8),

S. 609-617, http://www.boeckler.de/wsimit_2012_08_dietz.pdf

Fuchs, J./Söhnlein, D./Weber, B. (2011): Projektion des Arbeitskräfteangebots bis 2050. Rückgang und Alterung sind nicht mehr aufzuhalten, IAB-Kurzbericht 16/2011, Nürnberg

Klose, C./Bender, S. (2000): Berufliche Weiterbildung für Arbeitslose - ein Weg zurück in Beschäftigung?, in: MittAB 33 (3), S. 421-444

Koch, S./Spies, C./Stephan, G./Wolf, J. (2011): Arbeitsmarktinstrumente auf dem Prüfstand, IAB-Kurzbericht 11/2011, Nürnberg

Koller, L./Rudolph, H. (2011): Arbeitsaufnahme von SGB II Leistungsempfängern. Viele Jobs von kurzer Dauer, IAB-Kurzbericht 14/2011, Nürnberg

Mahlberg-Wilson, E./Mehlis, P./Quante-Brandt, E. (2009): Bleib dran... Sicherung des Ausbildungserfolgs durch Beratung und Vermittlung bei Konflikten in der dualen Berufsausbildung. Eine empirische Studie. Bremer Beiträge zur Praxisforschung, Akademie für Arbeit und Politik der Universität Bremen

Mehlis, P./Eberwein, W./Heibült, J. (2013): Arbeitsmarktintegration durch Weiterbildung. Die "Qualifizierungsoffensive" in der Hafenwirtschaft" als exemplarischer Fall, Berlin

Quante-Brandt, E. (2003): Konflikte im Spannungsfeld von Arbeit und Beruf, Chancen für die Gestaltung arbeitsorientierter Bildungsprozesse, Bielefeld Statistisches Bundesamt (2011): Weiterbildung, Wiesbaden

Witzel, A. (1989). Das problemzentrierte Interview, in: Jüttemann, G. (Hrsg.): Qualitative Forschung in der Psychologie. Grundfragen, Verfahrensweisen, Anwendungsfelder, S. 227-256, Heidelberg
Zentralverband der Deutschen Seehafenbetriebe (ZDS)/Bundesagentur für Arbeit/BMVBS (2007): Hafen- und Logistikbetriebe der deutschen Seehäfen starten Qualifizierungsoffensive für Langzeitarbeitslose, Pressemitteilung vom 7.12., Hamburg

\section{AUTOREN}

PETER MEHLIS, Dr. rer. pol., Wissenschaftlicher Mitarbeiter am Zentrum für Arbeit und Politik der Universität Bremen. Arbeitsschwerpunkte:Theorie und Praxis der betrieblichen Arbeitsbeziehungen, Arbeitszeit- und Beschäftigungspolitik, berufliche und politische Weiterbildung.

pmehlis@zap.uni-bremen.de

WILHELM EBERWEIN, Prof. Dr., (im Ruhestand), bis 2012 Lehre im Fachbereich Erziehungs- und Bildungswissenschaften der Universität Bremen. Arbeitsschwerpunkte: Soziologie und Entwicklung der industriellen Arbeit, Arbeitsbeziehungen/Arbeitsforschung.

eberwein@aap.uni-bremen.de

JESSICA HEIBÜLT, MA. Politologin, Wissenschaftliche Mitarbeiterin am Zentrum für Arbeit und Politik der Universität Bremen, Arbeitsschwerpunkte: Bildungsforschung und Arbeitsmarktpolitik.

jheibuelt@zap.uni-bremen.de 